\begin{tabular}{|c|c|}
\hline Title & High-temperature ultraviolet detection based on surface photovoltage effect in SiN passivated n-GaN films \\
\hline Author(s) & Matys, M.; A damowicz, B.; Zytkiewicz, Z. R.; Taube, A.; Kruszka, R.; Piotrowska, A . \\
\hline Citation & $\begin{array}{l}\text { A pplied physics letters, 109(5), } 051106 \\
\text { https://doi.org/10.1063/1.4960484 }\end{array}$ \\
\hline Issue Date & 2016-08-01 \\
\hline Doc URL & http:/hdl. handle.net/2115/66895 \\
\hline Rights & $\begin{array}{l}\text { This article may be downloaded for personal use only. Any other use requires prior permission of the author and AIP } \\
\text { Publishing. The following article appeared in A ppl. Phys. Lett. . 109, } 051106 \text { (2016) and may be found at } \\
\text { http:/scitation.aip.org/content/aip/ournal/apl/109/5/10.1063/1.4960484. }\end{array}$ \\
\hline Type & article \\
\hline File Information & 1.4960484.pdf \\
\hline
\end{tabular}

Instructions for use 


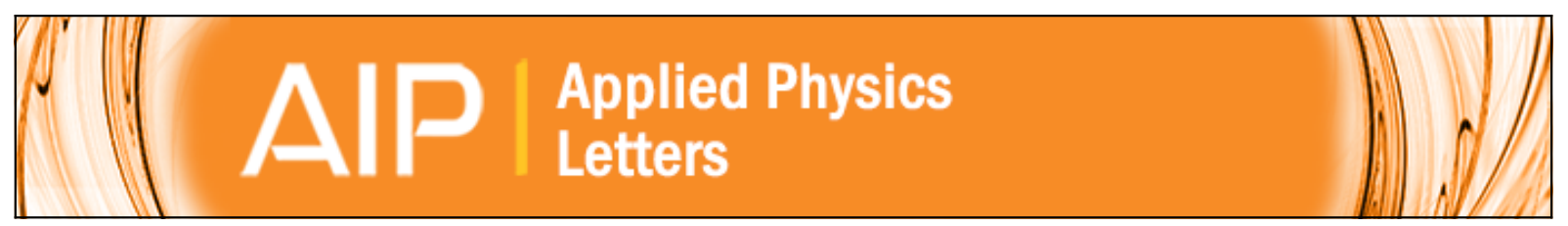

High-temperature ultraviolet detection based on surface photovoltage effect in SiN passivated $\mathbf{n}-\mathrm{GaN}$ films

M. Matys, B. Adamowicz, Z. R. Zytkiewicz, A. Taube, R. Kruszka, and A. Piotrowska

Citation: Applied Physics Letters 109, 051106 (2016); doi: 10.1063/1.4960484

View online: http://dx.doi.org/10.1063/1.4960484

View Table of Contents: http://scitation.aip.org/content/aip/journal/apl/109/5?ver=pdfcov

Published by the AIP Publishing

\section{Articles you may be interested in}

High-temperature and reliability performance of $4 \mathrm{H}-\mathrm{SiC}$ Schottky-barrier photodiodes for UV detection

J. Vac. Sci. Technol. B 33, 040602 (2015); 10.1116/1.4923083

Thermal stability and in situ SiN passivation of InAIN/GaN high electron mobility heterostructures

Appl. Phys. Lett. 105, 112101 (2014); 10.1063/1.4895807

Ultraviolet GaN photodetectors on Si via oxide buffer heterostructures with integrated short period oxide-based distributed Bragg reflectors and leakage suppressing metal-oxide-semiconductor contacts

J. Appl. Phys. 116, 083108 (2014); 10.1063/1.4894251

High-temperature ultraviolet detection based on InGaN Schottky photodiodes

Appl. Phys. Lett. 99, 031115 (2011); 10.1063/1.3615291

Very narrow-band ultraviolet photodetection based on strained M -plane GaN films

Appl. Phys. Lett. 90, 091110 (2007); 10.1063/1.2710769

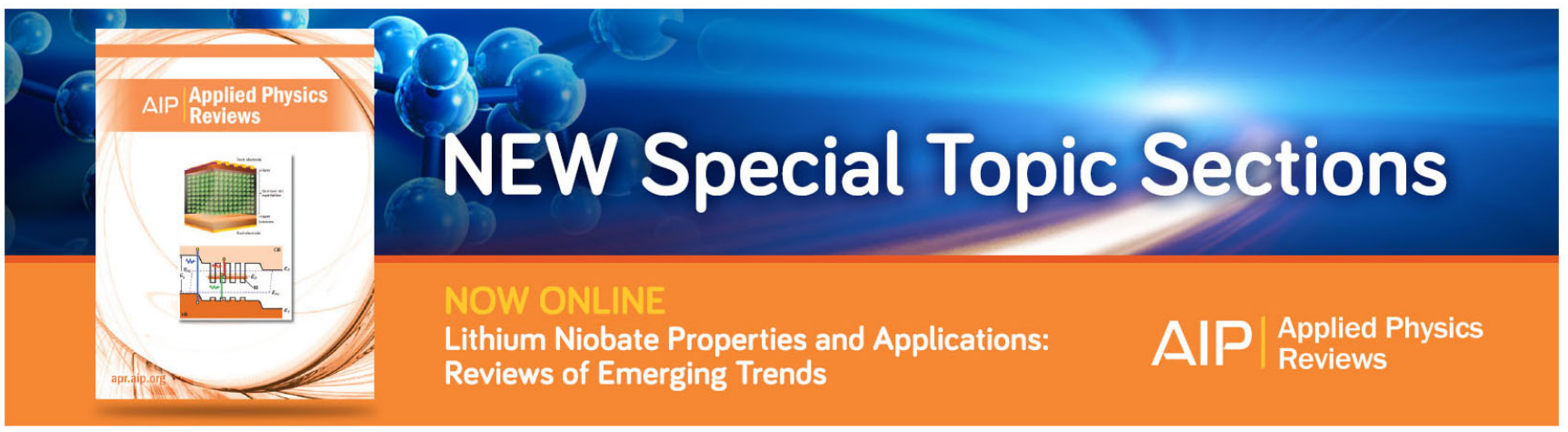




\title{
High-temperature ultraviolet detection based on surface photovoltage effect in SiN passivated $n-G a N$ films
}

\author{
M. Matys, ${ }^{1,2}$ B. Adamowicz, ${ }^{2}$ Z. R. Zytkiewicz, ${ }^{3}$ A. Taube,${ }^{4}$ R. Kruszka, ${ }^{4}$ and A. Piotrowska ${ }^{4}$ \\ ${ }^{1}$ Research Center for Integrated Quantum Electronics, Hokkaido University, Kita-13 Nishi-8, Kita-ku, \\ 060-8628 Sapporo, Japan \\ ${ }^{2}$ Surface Physics and Nanostructures Department, Institute of Physics-CND, \\ Silesian University of Technology, Konarskiego 22B, 44-100 Gliwice, Poland \\ ${ }^{3}$ Institute of Physics, Polish Academy of Sciences, al. Lotnikow 32/46, 02-668 Warsaw, Poland \\ ${ }^{4}$ Institute of Electron Technology, al. Lotnikow 32/46, 02-668 Warsaw, Poland
}

(Received 30 June 2016; accepted 25 July 2016; published online 5 August 2016)

\begin{abstract}
We investigated the surface photovoltage (SPV) effect in $\mathrm{n}-\mathrm{GaN}$ layers passivated with various insulators, i.e., $\mathrm{Al}_{2} \mathrm{O}_{3}, \mathrm{SiO}_{2}$, and $\mathrm{SiN}$ for ultraviolet (UV) light detection. We revealed that $\mathrm{SPV}$ in $\mathrm{SiN} / \mathrm{GaN}$ shows markedly different behaviour than in oxide/GaN, i.e., the photo-signal exhibited very fast response $(1 \mathrm{~s})$ and recovery $(2 \mathrm{~s})$ times, contrary to oxide/GaN, and it was thermally stable up to $523 \mathrm{~K}$. Furthermore, SPV spectra for SiN/GaN showed a sharp cut-off edge directly corresponding to the GaN band gap. We explained these results in terms of the different band structure of SiN/GaN and oxide/GaN junctions. All the observed properties of SPV response from SiN/GaN indicate that this relatively simple system can be applied to sensitive high temperature visible-blind UV detection. Published by AIP Publishing. [http://dx.doi.org/10.1063/1.4960484]
\end{abstract}

Ultraviolet (UV) sensitive $\mathrm{GaN}$ and $\mathrm{ZnO}$ based photodetectors working in the visible (VIS)-blind spectrum range have received significant attention in such fields like flame and missile plume detecting, chemical and biological agent sensing, and secured space-to-space communication and UV astronomy. ${ }^{1}$ In addition, in many cases UV photodetectors capable of operating at high temperatures (T) with high performance are greatly required. ${ }^{2}$ The conventional UV photodetector devices are usually based on $p-n$ and $p-i-n$ junctions, Schottky diodes, and metal-semiconductor-metal (MSM) structures. ${ }^{3,4}$ As a standard, such devices exhibit a good performance at room temperature (RT), i.e., fast response speed and low noise level. However, significant increase of dark current with $\mathrm{T}$ results in their limited practical applications at high $\mathrm{T}$. In order to avoid these drawbacks, metal-insulator-semiconductor (MIS) structures were adopted. ${ }^{5,6}$ Nevertheless, the fabrication process of high-T MIS type UV photodetectors is complicated and time-consuming, ${ }^{7}$ since it involves the deposition of high-quality thin dielectric films and lithography process. Thus including such devices in the practical routes seems to be difficult.

In this letter, we investigated the surface photovoltage (SPV) effect in $\mathrm{n}-\mathrm{GaN}$ layers passivated with various insulators, i.e., $\mathrm{Al}_{2} \mathrm{O}_{3}, \mathrm{SiO}_{2}$, and $\mathrm{SiN}$ in the context of UV detection. We found that in the case of SiN/GaN, SPV behaviour was significantly different and most advantageous for photodetecting applications compared to oxide/GaN structures. For example, the SPV signal in SiN/GaN exhibited very fast response/recovery times and high thermal stability up to $523 \mathrm{~K}$. We explained these features in terms of a model, in which the key role in SPV generation plays the band structure of the insulator/GaN junction. Furthermore, SPV spectra from $\mathrm{SiN} / \mathrm{GaN}$ exhibit a sharp cut-off edge which directly corresponds to the GaN band gap. All these properties of $\mathrm{SPV}$ in $\mathrm{SiN} / \mathrm{GaN}$ indicate that this relatively simple system can be applied to the sensitive high temperature VIS-blind UV detection.

In order to detect UV radiation based on SPV measurements, $\mathrm{n}-\mathrm{GaN}$ layers were passivated with various insulators $\left(\mathrm{Al}_{2} \mathrm{O}_{3}, \mathrm{SiO}_{2}, \mathrm{SiN}\right)$. The dielectric films were used to prevent the reactive $\mathrm{GaN}$ surface from the interaction with species in air ambient and uncontrolled formation of native oxides as well as to avoid the photo-induced adsorption/desorption effects under UV illumination. ${ }^{8}$ It should be noted that there is no reports on the SPV effect in GaN covered with dielectric films.

The schematic illustration of the examined GaN layers and SPV measurement system is presented in Fig. 1. In our studies we used GaN layers (thickness of $1 \mu \mathrm{m}$ ) obtained by

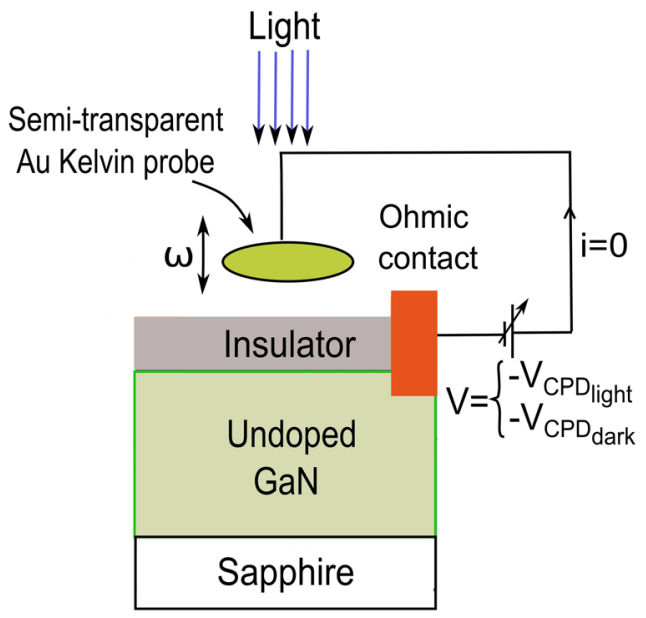

FIG. 1. Scheme of the examined GaN layers and the Kelvin probe system for SPV measurement. An Au semitransparent grid probe capacitively coupled with the sample vibrates due to the piezoelectric actuator in order to induce an ac current in the external circuit. The CPD is measured as the opposite of the dc voltage $\mathrm{V}$ (applied by the Kelvin probe controller) that nulls the ac current $(i=0)$. 
molecular beam epitaxy growth on sapphire substrates and intentionally undoped with a free electron concentration of about $10^{16} \mathrm{~cm}^{-3}$. The GaN layers were passivated with the dielectric films of: (i) $\mathrm{Al}_{2} \mathrm{O}_{3}$ (thickness of $63 \mathrm{~nm}$ ) prepared by atomic layer deposition (ALD), (ii) $\mathrm{SiO}_{2}(60 \mathrm{~nm})$ deposited by electron cyclotron resonance chemical vapor deposition (ECR CVD), and (iii) $\mathrm{SiN}(70 \mathrm{~nm})$ also deposited by ECR CVD. More details about the passivation process can be found in Refs. 9 and 10. Additionally, the ohmic contact was fabricated to ground the sample. The measurement of a SPV signal was performed using a vibrating semitransparent ring-shaped gold grid (diameter of $2 \mathrm{~mm}$ ) Kelvin probe (Besocke, type S) approached to the insulator film surface (distance of about $1 \mathrm{~mm}$ ) to obtain capacitance coupling with the sample. The Kelvin probe method allows to measure directly the contact potential difference (CPD) between the sample and gold grid in the dark $\left(V_{C P D_{\text {dark }}}\right)$ and upon illumination $\left(V_{C P D_{\text {light }}}\right)$. The measured SPV signal is the difference between $q V_{C P D_{\text {light }}}$ and $q V_{C P D_{\text {dark }}},{ }^{11}$ where $\mathrm{q}$ is the elementary charge. The light source was a $\mathrm{He}-\mathrm{Cd}$ laser with the wavelength $\lambda=325 \mathrm{~nm}$ (maximum power of $16 \mathrm{~mW}$ ) and an illuminator (from the infrared to UV range) equipped with a deuterium and halogen lamps. The SPV response was registered as a function of time ( $\mathrm{t}$ ) in a transient mode and in a steady-state mode as a function of the light intensity $(\Phi)$ and $\lambda$ at different $\mathrm{T}$, in air ambient.

Fig. 2 presents the light-on and light-off SPV transients for $\mathrm{Al}_{2} \mathrm{O}_{3} / \mathrm{GaN}, \mathrm{SiO}_{2} / \mathrm{GaN}$ and $\mathrm{SiN} / \mathrm{GaN}$ obtained under UV illumination (He-Cd laser) with $\Phi=10^{12}$ and $10^{17}$ photon/ $\left(\mathrm{cm}^{2} \mathrm{~s}\right)$ at $300 \mathrm{~K}, 423 \mathrm{~K}$, and $523 \mathrm{~K}$. One can note that the transient behaviour of SPV in the case of $\mathrm{Al}_{2} \mathrm{O}_{3} / \mathrm{GaN}$ and $\mathrm{SiO}_{2} / \mathrm{GaN}$ is very similar (Figs. 2(a) and 2(b)). After turn-on the UV light, the SPV signal, at low $\Phi$, raises slowly with t, whereas at higher $\Phi$, SPV increases more rapidly. After turnoff the UV illumination, we observe a very slow SPV decay in the case of low and high $\Phi$. On the other hand, we notice a markedly different SPV behaviour for SiN/GaN (Fig. 2(c)). For example, for both $\Phi$ values SPV raises rapidly after UV turn-on, requires short time to achieve the saturation and decays very fast after UV switch-off. The response and recovery times are around $1 \mathrm{~s}$ and $2 \mathrm{~s}$, respectively. It should be noted that the switching time was limited by the measurement time determined by the Kelvin probe system. We also noted that the SPV responses are well reproducible for both low and high $\Phi$ (Fig. 2(d)). Furthermore, the saturated value of SPV for SiN/GaN is thermally stable contrary to $\mathrm{Al}_{2} \mathrm{O}_{3} /$ $\mathrm{GaN}$ and $\mathrm{SiO}_{2} / \mathrm{GaN}$, for which the saturated SPV strongly decreased with increased $\mathrm{T}$.

In general, different transient decay times of SPV, as registered for oxide/GaN and SiN/GaN structures, should result from various interface properties, including the dark interface band bending $\left(q V_{S_{\text {dark }}}\right)$ and dynamical characteristics of the interface states in terms of the carrier capture cross section $(\sigma){ }^{11-13}$ The large $q V_{S_{\text {dark }}}$ is usually responsible for high SPV signal and long decay time whereas large $\sigma$ is responsible for short decay time. ${ }^{13}$ It should be noted that the interface properties in photodetecting structures can be properly modified by a suitable passivation for improving photo-responses, as it was reported by Liu and $\mathrm{Kim}^{14}$ for ZnO-based UV photodetectors.

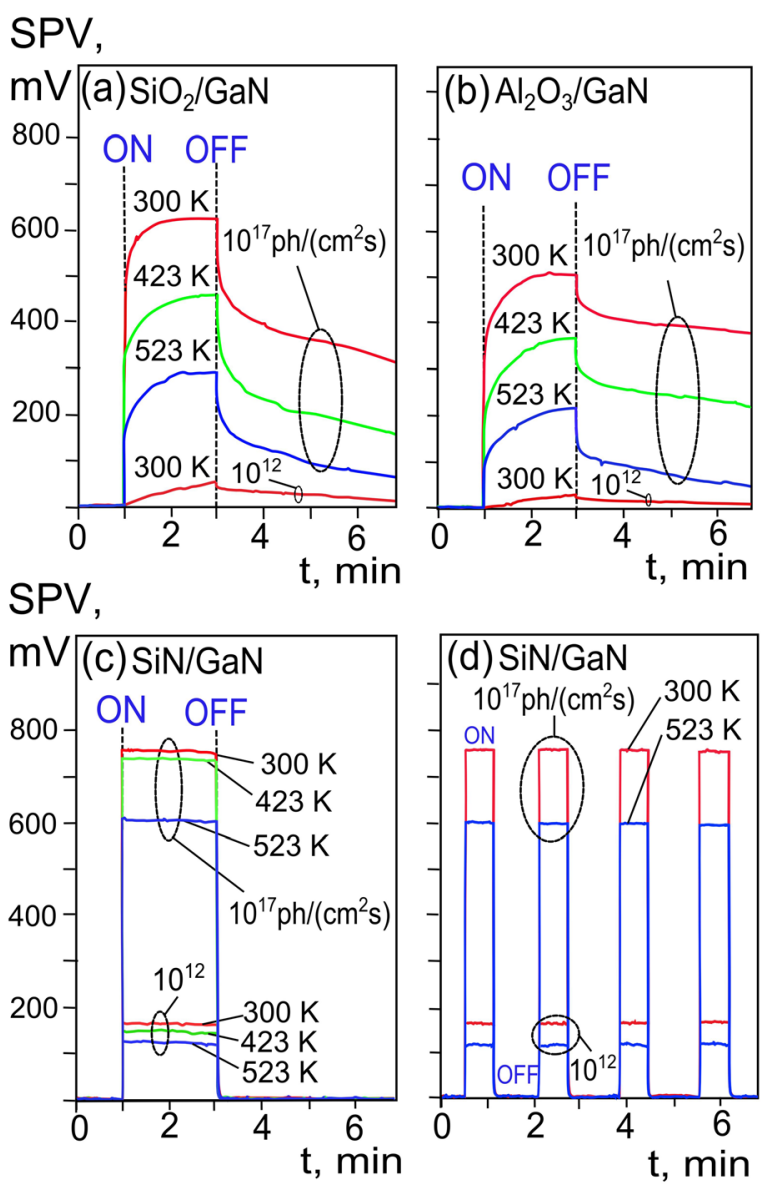

FIG. 2. Light-on and light-off SPV transients for $\mathrm{SiO}_{2} / \mathrm{GaN}$ (a), $\mathrm{Al}_{2} \mathrm{O}_{3} / \mathrm{GaN}$ (b) and $\mathrm{SiN} / \mathrm{GaN}$ (c) and cycles for SiN/GaN (d) obtained under UV illumination (He-Cd laser) with $\Phi=10^{12}$ and $10^{17}$ photon $/\left(\mathrm{cm}^{2} \mathrm{~s}\right)$ at $300 \mathrm{~K}$, $423 \mathrm{~K}$, and $523 \mathrm{~K}$.

In order to clarify the difference in SPV decays obtained for the oxide/GaN and $\mathrm{SiN} / \mathrm{GaN}$ structures, in terms of various interface characteristics, we used the band diagram in Fig. 3(a) illustrating an idea of the measurement of CPD and $\mathrm{SPV}$. One can find that the difference in $q V_{S_{\text {dark }}}$ between two samples can be expressed as

$$
q \triangle V_{S_{\text {dark }}}=q \triangle V_{C P D_{\text {dark }}}+q \triangle V_{I_{\text {dark }}},
$$

where $q \triangle V_{C P D_{\text {dark }}}$ is the difference of CPD between the samples in the dark and $q \triangle V_{I_{d a r k}}$ is the difference of the potential drop across insulators in the dark $\left(q V_{I_{\text {dark }}}\right)$. Because we found for the investigated $\mathrm{Al}_{2} \mathrm{O}_{3} / \mathrm{GaN}$ and $\mathrm{SiN} / \mathrm{GaN}$ structures that: (1) the measured $q \triangle V_{C P D_{\text {dark }}}$ is only about $50 \mathrm{meV}$ and (2) $q \triangle V_{I_{d a r k}}$ can be neglected (due to the similar values of the dielectric constant ${ }^{9}$ for $\mathrm{Al}_{2} \mathrm{O}_{3}$ and $\mathrm{SiN}$ layers and their similar thicknesses) thus we can conclude, using Eq. (1), that $q V_{S_{\text {dark }}}$ at $\mathrm{Al}_{2} \mathrm{O}_{3} / \mathrm{GaN}$ and $\mathrm{SiN} / \mathrm{GaN}$ interfaces should be comparable. Therefore, the difference in SPV transients for these structures should result mainly from various $\sigma$. On the other hand, our recent studies ${ }^{10}$ showed that $\sigma$ for SiN/III-N interface is lower more than one order of magnitude than for oxide/III-N interfaces. Therefore, the SPV decay time for SiN/GaN should be longer than for the oxidized GaN interface, which is in the evident contradiction with our observations. Thus, introducing an appropriate SPV mechanism to explain our observation is necessary. 


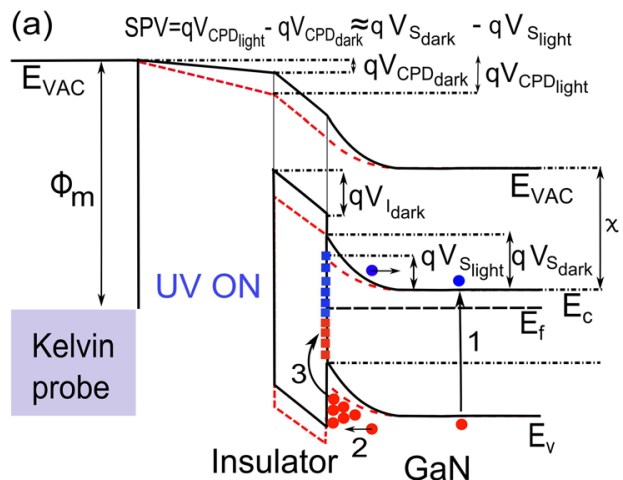

(b)

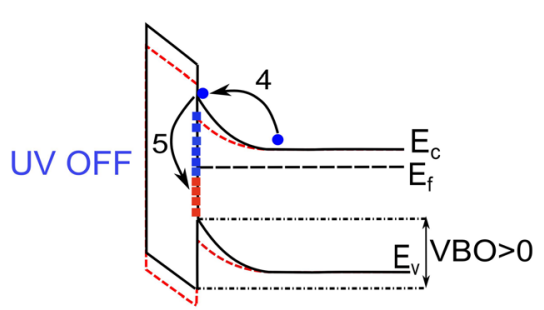

(c) $S P V=q V_{C P D_{\text {ight }}}-q V_{C P D_{\text {dark }}} q V_{l_{\text {dark }}}-q V_{\text {light }}$

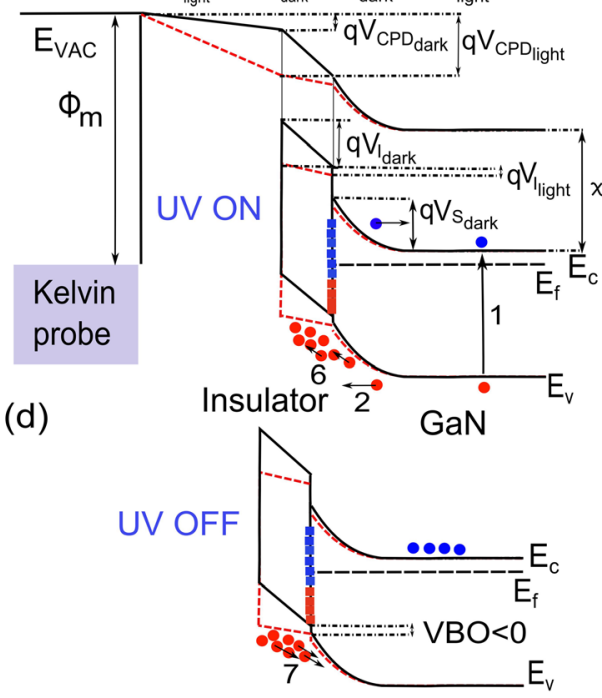

FIG. 3. Energy band diagrams explaining the principle of SPV effect for the insulator/GaN system with the positive (a) and (b) and small negative VBO (c) and (d) using the Kelvin probe. $E_{V A C}$ indicates the vacuum level, $E_{C}, E_{V}$ is the conduction band minimum and the valence band maximum, respectively, $\Phi_{m}$ is the Kelvin probe work function and $\chi$ is the electron affinity of $\mathrm{GaN}$. The black solid lines and red dashed lines illustrate the band diagrams under dark and illumination, respectively. Process (1) denotes the electronhole pair generation, (2) electron and hole drift in the depletion layer, (3) hole capture by the interface states, (4) and (5) thermionic emission of electrons and their capture by interface states, respectively, (6) and (7) flow of holes into the insulator and back after turn-off light, respectively.

The different transient decay time of SPV in oxide/GaN and $\mathrm{SiN} / \mathrm{GaN}$ can be explained in terms of the following model, which is schematically illustrated in Fig. 3. Within this model, the key role in SPV generation plays the band structure, in particular the valence band offset (VBO) between an insulator and GaN. According to most of the reports, $\mathrm{SiO}_{2}$ and $\mathrm{Al}_{2} \mathrm{O}_{3}$ have the significant positive $\mathrm{VBO}$ with respect to $\mathrm{GaN}$, i.e., for $\mathrm{SiO}_{2} \mathrm{VBO}$ is equal to $2 \mathrm{eV}$ (Ref. 15) and 3.2 eV (Ref. 16) and for $\mathrm{Al}_{2} \mathrm{O}_{3}$ lies between $0.7 \mathrm{eV}$ and $3.4 \mathrm{eV},{ }^{16-19}$ and only some authors indicate at almost negligible $\mathrm{VBO}$ for $\mathrm{Al}_{2} \mathrm{O}_{3} / \mathrm{GaN}^{20}$ On the other hand, most of the reports referring to $\mathrm{SiN} / \mathrm{GaN}$ claim that $\mathrm{VBO}$ is slightly negative ${ }^{21,22}$ and only some works give positive VBO of about $0.8 \mathrm{eV}$ (Ref. 16) for this system. On this basis, we assumed that the studied $\mathrm{Al}_{2} \mathrm{O}_{3} / \mathrm{GaN}$ and $\mathrm{SiO}_{2} / \mathrm{GaN}$ have the positive $\mathrm{VBO}$ and $\mathrm{SiN} / \mathrm{GaN}$ has a small negative VBO.

Upon UV illumination, the excess carriers are generated in $\mathrm{GaN}$ and become quickly separated in the depletion layer, i.e., electrons are repelled into the bulk whereas holes are attracted towards the interface. In the case of $\mathrm{Al}_{2} \mathrm{O}_{3}$ and $\mathrm{SiO}_{2}$, the excess holes are collected near the insulator/GaN interface and subsequently captured by donor-like interface states, as shown in Fig. 3(a). This causes partial compensation of the negative charge at the insulator/GaN interface and reduction of the interface band bending from $q V_{S_{\text {dark }}}$ to $q V_{S_{\text {light }}}$. As a consequence, the SPV signal, which corresponds to the difference between $q V_{S_{\text {dark }}}$ and $q V_{S_{\text {light }}}$, is generated. After switching off the light (Fig. 3(b)), the interface states become slowly filled by electrons due to their thermionic emission ${ }^{12}$ from the bulk over the barrier. As a result, the interface band bending recovers and SPV signal slowly decays. In the case of SiN, only a small part of the generated photo-holes is captured by the interface states while the remaining holes flow into the insulator layer, as shown in Fig. 3(c). This causes the marked change in the potential drop within the insulator layer from $q V_{I_{\text {dark }}}$ to $q V_{I_{\text {light }}}$ and rather slight variation in the interface band bending. As a result, the induced SPV signal mainly corresponds to the difference between $q V_{I_{d a r k}}$ and $q V_{I_{l i g h t}}$. After turning off the light (Fig. 3(d)), the excess holes quickly leave the insulator and recombine with bulk electrons in $\mathrm{GaN}$, which leads to the potential recovery in the insulator and fast decay of SPV.

From Figs. 2(c) and 2(d) it follows that $\mathrm{SiN} / \mathrm{GaN}$ seems to be the best candidate for UV light detection. In order to confirm this, we performed the measurement of a steadystate SPV response as a function of $\lambda$ and $\Phi$ at $300 \mathrm{~K}$ and $523 \mathrm{~K}$. The obtained SPV spectra, summarized in Fig. 4(a), exhibit a sharp cut-off edge $\left(\lambda_{\text {GaN }}\right)$, which directly corresponds to the GaN band gap. One can also note that for $\lambda<\lambda_{\text {GaN }}$ the SPV signal is rather stable whereas for $\lambda>\lambda_{\text {GaN }}$ totally vanishes. On the other hand, the SPV $(\Phi)$ curves exhibit a linear dependence up to $\Phi_{0}=1 \times 10^{11}$ photon $/\left(\mathrm{cm}^{2} \mathrm{~s}\right)$ as shown in Fig. 4(b) and logarithmic one above $\Phi_{0}$, as in Fig. 4(c). The similar two ranges of SPV were reported in Refs. 11, 12, 23, and 24. In addition, the SPV response at both $\mathrm{T}$ can be detected at very low $\Phi \leq 5 \times 10^{10} \mathrm{photon} /\left(\mathrm{cm}^{2} \mathrm{~s}\right)$ $\left(\approx 0.03 \mu \mathrm{W} / \mathrm{cm}^{2}\right)$. All the observed properties of $\mathrm{SPV}$ response from $\mathrm{SiN} / \mathrm{GaN}$ indicate that this relatively simple system can be applied to sensitive VIS-blind UV detection at various radiation ranges and at very high $\mathrm{T}$ up to $523 \mathrm{~K}$. In order to achieve a simpler and convenient device construction, the vibrating Kelvin probe arrangement can be easily substituted by a system with the constant capacitor (with a UV-transparent conducting film deposited on an insulator surface) and modulated light or by the introducing an alternating current source in the electric circuit for the detection of the null voltage condition to determine the CPD value. ${ }^{25}$

In conclusion, we studied the SPV effect in $n-G a N$ layers passivated with different insulators, i.e., $\mathrm{Al}_{2} \mathrm{O}_{3}, \mathrm{SiO}_{2}$, and $\mathrm{SiN}$. We revealed that $\mathrm{SPV}$ in $\mathrm{SiN} / \mathrm{GaN}$ shows markedly different behaviour than in oxide/GaN. For example, in the case of SiN/GaN, the SPV signal exhibited very fast 
(a)
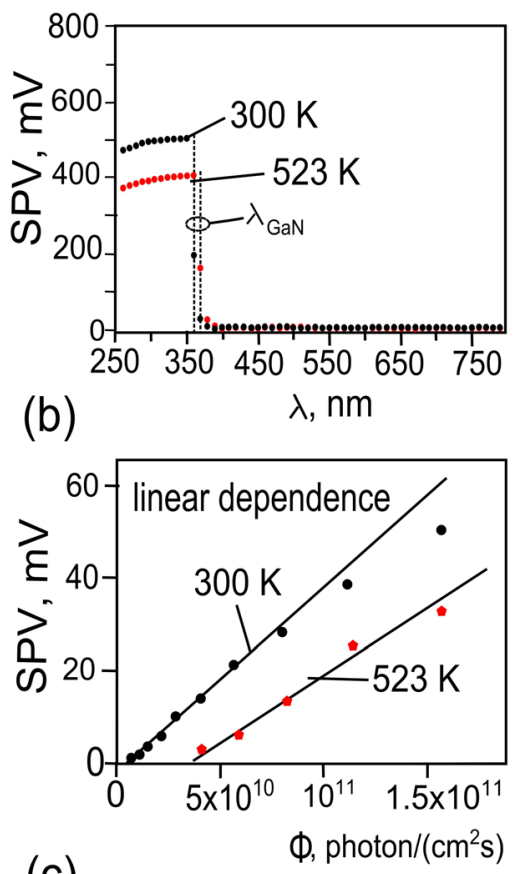

(c)

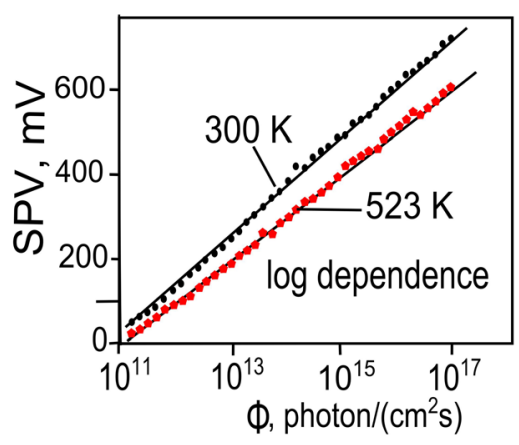

FIG. 4. SPV spectra from $\mathrm{SiN} / \mathrm{GaN}$ obtained at $\Phi=10^{15}$ photon $/\left(\mathrm{cm}^{2} \mathrm{~s}\right)$ using a deuterium/halogen illuminator (a); $\operatorname{SPV}(\Phi)$ dependences in the linear (b) and logarithmic regimes (c) obtained using a He-Cd laser; measurements at $300 \mathrm{~K}$ and $523 \mathrm{~K}$.

response/recovery times, contrary to oxide/GaN, and it was thermally stable up to $523 \mathrm{~K}$. We explained these results in terms of the different band structure of SiN/GaN and oxide/ GaN junctions. Furthermore, we found that SPV spectra for $\mathrm{SiN} / \mathrm{GaN}$ showed a sharp cut-off edge directly corresponding to the GaN band gap. All the observed properties of SPV response of $\mathrm{SiN} / \mathrm{GaN}$ indicate that this relatively simple system can be applied to sensitive high-temperature VIS-blind UV detection. Thus, our work can provide a route for developing high-temperature and high-performance UV photodetectors.

One of the authors, M.M. thanks RCIQE, Hokkaido University, Sapporo, Japan for the postdoctoral fellowship and the members of RCIQE for their kind assistance and discussion. The work was partially supported by the InTechFun Project (UDA-POIG.01.03.01.00-159/08-04) of EU Structural Funds in Poland and the Visegrad Group (V4)-Japan Joint Research Program on Advanced Materials, SAFEMOST project (14/990/PNN16/0072).

${ }^{1}$ UV Solid-State Emitters and Detectors, NATO Science Series II: Mathematics, Physics and Chemistry, edited by M. S. Shur and A. Zukauskas (Kluwer Academic, Norwell, Massachusetts, 2004).

${ }^{2}$ H. Y. Chen, K. W. Liu, L. F. Hu, A. A. Al-Ghamdi, and X. S. Fang, Mater. Today 18, 493 (2015).

${ }^{3}$ M. Razeghi and A. Rogalski, J. Appl. Phys. 79, 7433 (1996).

${ }^{4}$ C. Rivera, J. Pereiro, A. Navarro, E. Munoz, O. Brandt, and H. T. Grahn, Open Electr. Electron. Eng. J. 4, 1 (2010).

${ }^{5}$ C. Mizue, M. Miczek, J. Kotani, and T. Hashizume, Jpn. J. Appl. Phys. 48, 020201 (2009).

${ }^{6}$ L. W. Sang, M. Y. Liao, Y. Koide, and M. Sumiya, Appl. Phys. Lett. 99, 031115 (2011).

${ }^{7}$ R. Zou, Z. Zhang, Q. Liu, J. Hu, L. Sang, M. Liao, and W. Zhang, Small 10, 1848 (2014).

${ }^{8}$ M. Foussekis, A. A. Baski, and M. A. Reshchikov, Appl. Phys. Lett. 94, 162116 (2009).

${ }^{9}$ C. Mizue, Y. Hori, M. Miczek, and T. Hashizume, Jpn. J. Appl. Phys., Part 1 50, 021001 (2011).

${ }^{10}$ M. Matys, R. Stoklas, J. Kuzmik, B. Adamowicz, Z. Yatabe, and T. Hashizume, J. Appl. Phys. 119, 205304 (2016).

${ }^{11}$ L. Kronik and Y. Shapira, Surf. Sci. Rep. 37, 1 (1999).

${ }^{12}$ M. A. Reshchikov, M. Foussekis, and A. A. Baski, J. Appl. Phys. 107, 113535 (2010).

${ }^{13}$ M. A. Reshchikov, S. Sabuktagin, D. K. Johnstone, and H. Morko, J. Appl. Phys. 96, 2556 (2004).

${ }^{14}$ M. Liu and H. K. Kim, Appl. Phys. Lett. 84, 173 (2004).

${ }^{15}$ T. E. Cook, C. C. Fulton, W. J. Mecouch, K. M. Tracy, R. F. Davis, E. H. Hurt, G. Lucovsky, and R. J. Nemanich, J. Appl. Phys. 93, 3995 (2003).

${ }^{16}$ J. Robertson and B. Falabretti, J. Appl. Phys. 100, 014111 (2006).

${ }^{17}$ L. Duan, J. S. Pan, and D. S. Ang, Appl. Phys. Lett. 102, 201604 (2013).

${ }^{18}$ Z. Zhang, C. M. Jackson, A. R. Arehart, B. McSkimming, J. S. Speck, and S. A. Ringel, J. Electron. Mater. 43, 828 (2014).

${ }^{19}$ J. Yang, B. S. Eller, C. Zhu, C. England, and R. J. Nemanich, J. Appl. Phys. 112, 053710 (2012).

${ }^{20}$ M. R. Coan, J. H. Woo, D. Johnson, I. R. Gatabi, and H. R. Harris, J. Appl. Phys. 112, 024508 (2012).

${ }^{21}$ T. E. Cook, Jr., C. C. Fulton, W. J. Mecouch, R. F. Davis, G. Lucovsky, and R. J. Nemanich, J. Appl. Phys. 94, 3949 (2003).

${ }^{22}$ M. Kumar, B. Roul, T. N. Bhat, M. K. Rajpalke, A. T. Kalghatgi, and S. B. Krupanidhi, Thin Solid Films 520, 4911 (2012).

${ }^{23}$ B. Adamowicz and H. Hasegawa, Vacuum 54, 173 (1999).

${ }^{24}$ M. Matys, P. Powroznik, D. Kupka, and B. Adamowicz, Opt. Appl. 43, 47 (2013).

${ }^{25}$ A. Henning, G. Gnzburger, R. Jhr, Y. Rosenwaks, B. Bozic-Weber, C. E. Housecroft, E. C. Constable, E. Meyer, and T. Glatzel, Beilstein J. Nanotechnol. 4, 418 (2013). 